\title{
Fission product yield measurements using monoenergetic photon beams
}

\author{
Krishichayan $^{1,2, a}$, M. Bhike ${ }^{1,2}$, A.P. Tonchev ${ }^{3}$, and W. Tornow ${ }^{1,2}$ \\ 1 Department of Physics, Duke University, Durham, NC 27708, USA \\ 2 Triangle Universities Nuclear Laboratory, Durham NC 27708, USA \\ 3 Nuclear and Chemical Sciences Division, Lawrence Livermore National Laboratory, Livermore, CA 94550, USA
}

\begin{abstract}
Measurements of fission products yields (FPYs) are an important source of information on the fission process. During the past couple of years, a TUNL-LANL-LLNL collaboration has provided data on the FPYs from quasi monoenergetic neutron-induced fission on ${ }^{235} \mathrm{U},{ }^{238} \mathrm{U}$, and ${ }^{239} \mathrm{Pu}$ and has revealed an unexpected energy dependence of both asymmetric fission fragments at energies below $4 \mathrm{MeV}$. This peculiar FPY energy dependence was more pronounced in neutron-induced fission of ${ }^{239} \mathrm{Pu}$. In an effort to understand and compare the effect of the incoming probe on the FPY distribution, we have carried out monoenergetic photon-induced fission experiments on the same ${ }^{235} \mathrm{U},{ }^{238} \mathrm{U}$, and ${ }^{239} \mathrm{Pu}$ targets. Monoenergetic photon beams of $E_{\gamma}=13.0 \mathrm{MeV}$ were provided by the $\mathrm{HI} \gamma \mathrm{S}$ facility, the world's most intense $\gamma$-ray source. In order to determine the total number of fission events, a dual-fission chamber was used during the irradiation. These irradiated samples were counted at the TUNL's low-background $\gamma$-ray counting facility using high efficient HPGe detectors over a period of 10 weeks. Here we report on our first ever photofission product yield measurements obtained with monoenegetic photon beams. These results are compared with neutron-induced FPY data.
\end{abstract}

\section{Introduction}

Nuclear fission is a highly complicated nuclear reaction phenomenon. An understanding of observables resulting from nuclear fission requires information on both static nuclear properties and large-scale nuclear dynamics, as well as understanding of nuclear configurations in extreme conditions, and the respective strengths of collective and single-particle degrees of freedom [1]. The process of nuclear fission starts with the formation of a compound nucleus which could be produced through a variety of excitation mechanisms, each with its own peculiarities. Particle-bombardment is one such means and tends to produce an initial nucleus with high angular momentum and excitation energy. For this reason, particle-induced fission is involves high angular momentum. The use of real photons, on the other hand, preferentially produces low-spin configurations at all energies. The benefit of a low spin is that the complexity of the reaction is reduced. Extraction of information from the reaction is, therefore, less complicated and can be potentially understood better. Fission induced by photons, photofission, is therefore a useful method for investigating the low-energy regime, which is the most important one for practical applications.

Fission-product yields (FPYs) have historically been one of the most studied observables of fission. A joint collaboration between TUNL, LANL, and LLNL has recently provided data on the energy dependence of high-yield Fission products from monoenergetic neutroninduced fission of ${ }^{235} \mathrm{U},{ }^{238} \mathrm{U}$, and ${ }^{239} \mathrm{Pu}$ between 0.5 and $14.8 \mathrm{MeV}$ [3]. According to this work [3], unlike

a e-mail: krishi@tunl.duke.edu; krishichayan@gmail.com
${ }^{235} \mathrm{U}$ and ${ }^{238} \mathrm{U}$, the cumulative FPYs obtained for ${ }^{239} \mathrm{Pu}$ show an unexpected energy dependence for certain highyield fission products like ${ }^{99} \mathrm{Mo},{ }^{140} \mathrm{Ba}$, and ${ }^{147} \mathrm{Nd}$. This is an important finding because it demonstrates that the high-yield fission products can have a dependence on incident neutron energy. As of now, there is a scarcity of photofission data obtained with monoenergetic photons. In an effort to understand and compare the effect of the incoming probe, especially the energy dependence, a program has been initiated at TUNL to carry out a systematic study of photofission with monoenergetic photon beams. In the present work, we report our preliminary results on FPY measurements on ${ }^{235} \mathrm{U},{ }^{238} \mathrm{U}$, and ${ }^{239} \mathrm{Pu}$ carried out using $13 \mathrm{MeV}$ photon beams provided by the High Intensity $\gamma$-ray Source $(\mathrm{HI} \gamma \mathrm{S})$ facility at TUNL.

\section{Experimental procedure, analysis, and results}

$\mathrm{HI} \gamma \mathrm{S}$, located on the campus of Duke University, generated circularly polarized and monoenergetic photon beams for the present experiment. Details on the $\mathrm{HI} \gamma \mathrm{S}$ facility can be found in Ref. [4] and are briefly summarized here. The $\mathrm{HI} \gamma \mathrm{S}$ facility produces a quasi-monoenergetic Compton backscattered photon beam tunable in wide energy range with either linear or circular polarization. A high-flux photon beam is created by colliding relativistic electron beam bunches, circulating in a storage ring, with photon pulses inside the high-power optical cavity of a free-electron laser (FEL). The peak energy of the photon beam was selected by an Al collimator of length $44.5 \mathrm{~cm}$ 


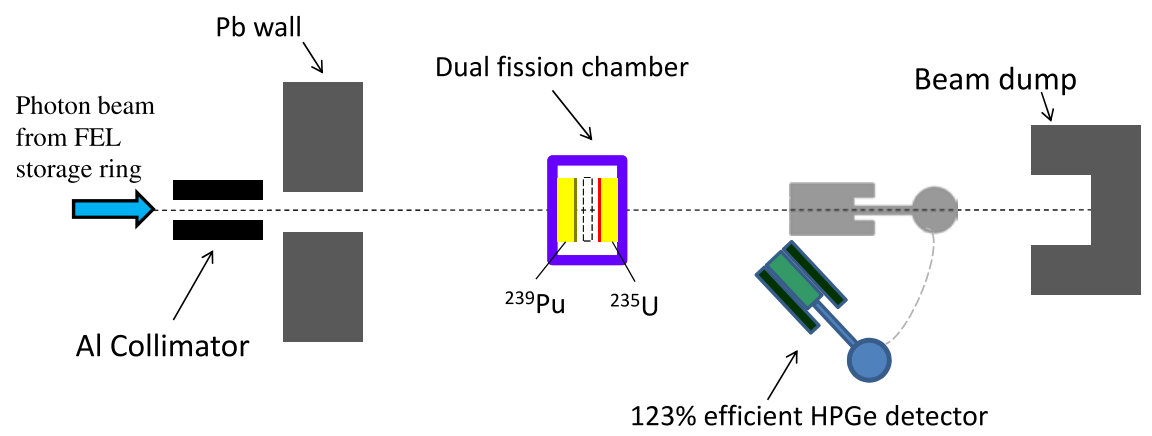

Figure 1. Schematic diagram (not to scale) of the experimental setup using a dual-fission chamber and a $123 \%$ efficient (relative) HPGe detector.

with a cylindrical hole of $1.27 \mathrm{~cm}$ diameter and positioned 55 meters downstream from the FEL photon-electron collision point in the storage ring. This collimator diameter results in an energy spread of $\sim 2-3 \%$ (FWHM) of the photon beam. Aluminum was chosen as the collimator material to minimize the number of neutrons produced via $(\gamma, \mathrm{n})$ reaction because of its high neutron separation energy of $13.6 \mathrm{MeV}$.

The principal task in obtaining accurate FPY data is the precise determination of the number of fissions which occur during the measurements. To accomplish this, a specially fabricated dual-fission ionization chamber (DFC) was used, as seen in the present experimental setup (Fig. 1). Such fission chambers have extensively been used in our neutron-induced FPY measurements. Details of a dual-fission chamber can be found in Refs. [2,3] and they are briefly summarized here. The dual-fission chamber was adopted from the design developed by Gilliam et al. at NIST [5]. However, several design changes were made to facilitate the insertion and removal of the activation target contained between the two individual fission chambers (FCs). The gas connections to the individual FCs are made of stainless steel tubing. The signals from each of the FCs are sent to the manifold via a UT-085 semirigid coaxial cable that runs inside the gas line. P-10 (90\% argon and 10\% methane) was used as the DFC fill gas. Signals from the FCs are input to Ortec 142-PC preamplifiers. The preamplified signals are then amplified by a Canberra 2026 spectroscopic amplifier and then fed to a Canberra Multiport II multichannel analyzer, supported by the GENIE2000 software [6].

For the present photon-induced measurement, two thin monitor foils $\left({ }^{235} \mathrm{U}\right.$ in chamber 1 and ${ }^{239} \mathrm{Pu}$ in chamber 2) were used. The ${ }^{239} \mathrm{Pu}$ activation target was placed at the center of the fission chamber and two other actinide samples were placed at the downstream end of the fission chamber. The DFC was placed at a distance of $\sim 58 \mathrm{~m}$ from the FEL collision point, which eliminates the need for a source-target angular-correlation correction, because the same collimated photon flux is seen by both chambers. Table 1 gives detailed information about the reference foils and activation targets used in the present work. The mass of the reference foils was determined by alpha spectroscopy using well calibrated ionization chambers at LANL. The mass measurement of each foil is given the conservative uncertainty of $\pm 1 \%$.

A $123 \%$ efficient (relative to a standard $7.62 \mathrm{~cm} \times$ $7.62 \mathrm{~cm} \mathrm{NaI}$ detector) coaxial HPGe detector was placed downstream of the fission chamber position to
Table 1. Information on the reference foils and activation targets used in the present experiment. Diameter of each reference foil and activation target is $1.27 \mathrm{~cm}$.

\begin{tabular}{llll}
\hline \hline $\begin{array}{l}\text { Monitor } \\
\text { Foil }\end{array}$ & Chamber & $\begin{array}{l}\text { Mass } \\
(\mu \mathrm{g})\end{array}$ & $\begin{array}{l}\text { Isotopic } \\
\text { abundance(\%) }\end{array}$ \\
\hline${ }^{235} \mathrm{U}$ & FC1 & $\begin{array}{l}160.36(160) \\
8.52(3)\end{array}$ & $\begin{array}{l}99.835(2) \\
99.954(2)\end{array}$ \\
\hline${ }^{239} \mathrm{Pu}$ & FC2 & & \\
\hline & & & \\
\hline Activation & Mass & Isotopic & \\
target & $(\mathrm{mg})$ & abundance(\%) & \\
\hline${ }^{235} \mathrm{U}$ & $223.02(2)$ & $93.27(3)$ & \\
${ }^{238} \mathrm{U}$ & $236.6(6)$ & $99.70(8)$ & \\
${ }^{239} \mathrm{Pu}$ & $233.0(2)$ & $98.41(40)$ & \\
\hline \hline
\end{tabular}

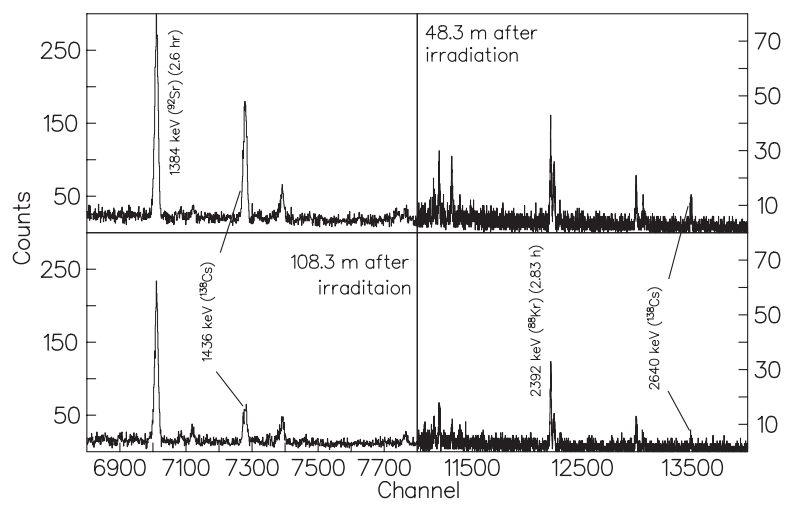

Figure 2. Spectra showing $\gamma$-rays from the decay of the fission fragment ${ }^{138} \mathrm{Cs}$ (half-life $=33.41$ minutes). The upper panel shows the data collected 48.3 minutes after the end of irradiation of ${ }^{238} \mathrm{U}$, whereas the lower panel gives the data taken 108.3 minutes after the end of irradiation.

measure the beam-energy distribution. During beamenergy measurements, the photon beam was attenuated by a series of copper attenuators mounted upstream.

After 62 hours of continuous irradiation with $\sim 10^{8} \gamma / \mathrm{s}$, the target samples $\left({ }^{235} \mathrm{U},{ }^{238} \mathrm{U}\right.$, and $\left.{ }^{239} \mathrm{Pu}\right)$ were $\gamma$-ray counted in TUNL's low-background counting facility using high-efficient HPGe detectors over a period of 2 months. Spectra were accumulated using the GENIE2000 system [6]. Offline $\gamma$-ray analysis was done using the RADWARE [7] and TV [8] gamma-analysis software. A typical $\gamma$-ray spectrum for the ${ }^{238} \mathrm{U}$ target is shown in Fig. 2, where decay $\gamma$-rays are clearly seen for the ${ }^{138} \mathrm{Cs}$ fission fragment, having a half-life of 33.41 minutes. These decay spectra were used to uniquely identify the products and to ensure that no 


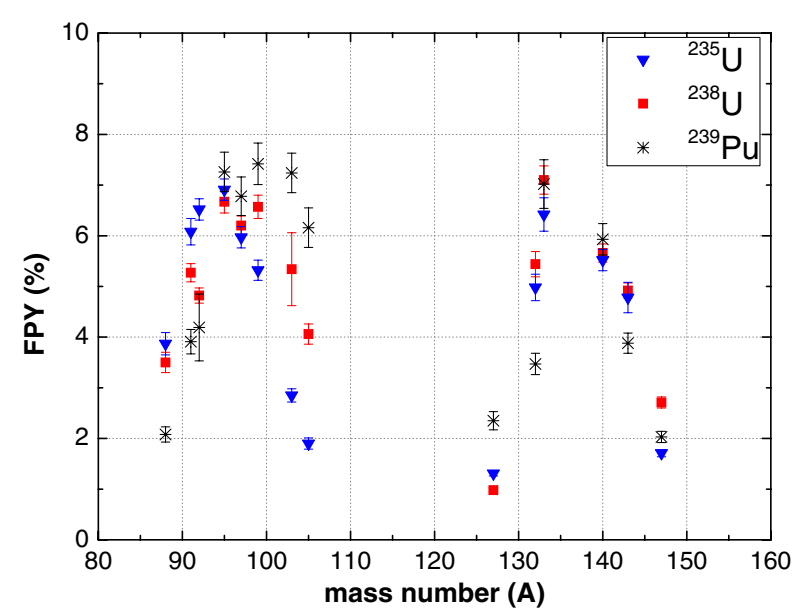

Figure 3. Plot of the mass-yield distributions measured in the present photofission of ${ }^{235} \mathrm{U},{ }^{238} \mathrm{U}$, and ${ }^{239} \mathrm{Pu}$.

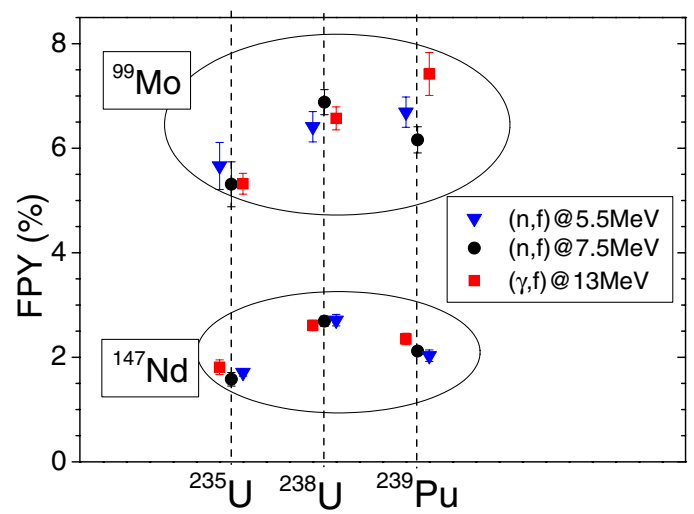

Figure 4. Fission-product yields from the present measurement for ${ }^{99} \mathrm{Mo}$ and ${ }^{147} \mathrm{Nd}$ are compared with neutron-induced FPY values.

interfering transitions were present. Several fragments, such as ${ }^{135} \mathrm{Xe}$ or ${ }^{140} \mathrm{La}$, are produced directly from fission and as daughter nuclei from other fission product parents with similar or longer half-lives. In such cases, fitting and deconvoluting the decay curves is necessary to determine the number of nuclei produced at $\mathrm{t}=0$ (end of activation). Extensive background measurements were performed prior to irradiation in identical geometrical conditions as the counting of the activated targets to check on interferences in the region of interest.

More than 30 fission fragments were observed and absolute cumulative fission product yields for these isotopes were determined, closely following the procedure described in detail in Ref. [3]. A major part of the error in this measurement comes from errors associated with the detector efficiency and photo-peak area analysis. The preliminary results of cumulative fission product yields for 14 fission fragments are tabulated in Table 2. Massyield distributions of photofission of ${ }^{235} \mathrm{U},{ }^{238} \mathrm{U}$, and ${ }^{239} \mathrm{Pu}$ are shown in Fig. 3. Figure 4 presents the comparison of fission-product yields from the present measurements for two high-yield isotopes $\left({ }^{99} \mathrm{Mo}\right.$ and $\left.{ }^{147} \mathrm{Nd}\right)$ with the values obtained in 5.5 and $7.5 \mathrm{MeV}$ neutron-induced FPY measurements [3]. The photon-induced FPY values for both isotopes are very close to the neutron-induced data.
Table 2. Partial list of fission-product yields (in \%) from $13 \mathrm{MeV}$ monoenergetic photon induced fission of ${ }^{235} \mathrm{U},{ }^{238} \mathrm{U}$, and ${ }^{239} \mathrm{Pu}$.

\begin{tabular}{llll}
\hline \hline $\begin{array}{l}\text { Fission } \\
\text { product }\end{array}$ & ${ }^{235} \mathrm{U}$ & ${ }^{238} \mathrm{U}$ & ${ }^{239} \mathrm{Pu}$ \\
\hline${ }^{88} \mathrm{Kr}$ & $3.87(22)$ & $3.50(20)$ & $2.08(15)$ \\
${ }^{91} \mathrm{Sr}$ & $6.08(26)$ & $5.27(18)$ & $3.91(24)$ \\
${ }^{92} \mathrm{Sr}$ & $6.52(21)$ & $4.82(15)$ & $4.19(66)$ \\
${ }^{95} \mathrm{Zr}$ & $6.91(21)$ & $6.67(22)$ & $7.26(39)$ \\
${ }^{97} \mathrm{Zr}$ & $5.97(21)$ & $6.20(19)$ & $6.78(38)$ \\
${ }^{99} \mathrm{Mo}$ & $5.32(20)$ & $6.57(22)$ & $7.42(41)$ \\
${ }^{103} \mathrm{Ru}$ & $2.85(13)$ & $5.34(72)$ & $7.24(39)$ \\
${ }^{105} \mathrm{Ru}$ & $1.90(11)$ & $4.06(20)$ & $6.16(39)$ \\
${ }^{127} \mathrm{Sb}$ & $1.31(5)$ & $0.98(04)$ & $2.35(18)$ \\
${ }^{132} \mathrm{Te}$ & $4.98(26)$ & $5.44(25)$ & $3.47(21)$ \\
${ }^{133} \mathrm{I}$ & $6.42(33)$ & $7.10(28)$ & $7.02(48)$ \\
${ }^{140} \mathrm{Ba}$ & $5.52(21)$ & $5.66(18)$ & $5.93(31)$ \\
${ }^{143} \mathrm{Ce}$ & $4.78(30)$ & $4.92(15)$ & $3.88(20)$ \\
${ }^{147} \mathrm{Nd}$ & $1.71(7)$ & $2.71(11)$ & $2.03(11)$ \\
\hline \hline
\end{tabular}

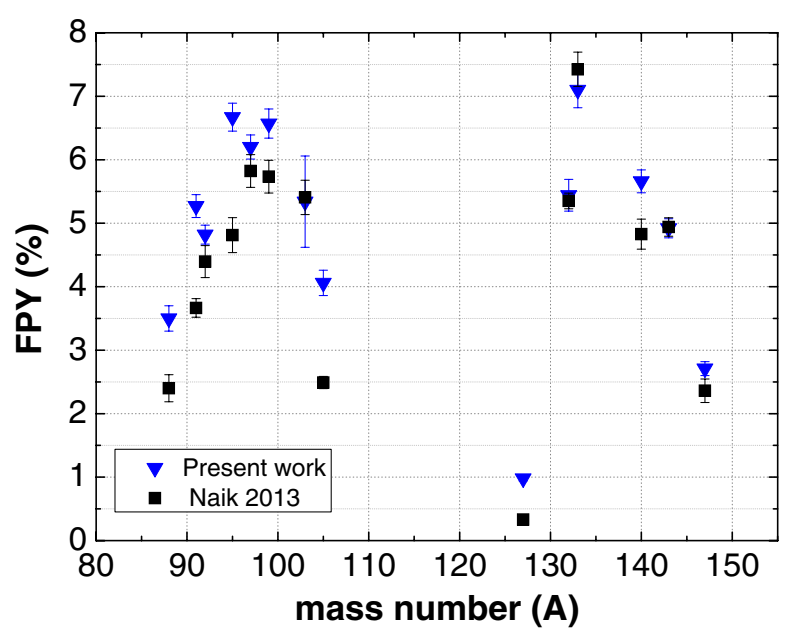

Figure 5. (Color Online) Mass-yield distributions of 14 fission fragments in $13.4 \mathrm{MeV}$ end-point bremsstrahlung-induced fission of ${ }^{238} \mathrm{U}$ [9] are compared with our present data obtained with monoenergetic photons at $13.0 \mathrm{MeV}$.

In 2013, Naik et al. [9] studied mass-yield distributions of fission products from photofission of ${ }^{238} \mathrm{U}$ induced by 11.5-17.3 MeV bremsstrahlung. Figure 5 compares our $13 \mathrm{MeV}$ monoeneregtic photofission data with $13.4 \mathrm{MeV}$ end-point bremsstrahlung-induced fission data of ${ }^{238} U$ [9]. As can be seen, the FPYs for almost all of the fragments of ${ }^{238} \mathrm{U}$ are consistently lower than our data.

In the near future, data will be obtained at lower and higher photon energy to study the effect of the incoming probe on FPYs and on the energy dependence of important high-yield fission products.

This work was supported partially by the US Department of Energy, Office of Nuclear Physics, under Grant No. DEFG02-97ER41033, and by the National Nuclear Security Administration under the Stewardship Science Academic Alliance Program through the US Department of Energy, Grant No. DE-NA0002793 and performed under the auspices of Lawrence Livermore National Security, LLC, (LLNS) under contract No. DEAC52-07NA27334. 


\section{References}

[1] H. Goutte, J.F. Berger, P. Casoli, D. Gogny, Phys. Rev. C 71, 024316 (2005)

[2] C. Bhatia et al., Nuclear Instruments and Methods in Physics Research Section A 757, 7 (2013)

[3] M.E. Gooden, C.W. Arnold, J.A. Becker, C. Bhatia, M. Bhike, E.M. Bond, T.A. Bredeweg, B. Fallin, M.M. Fowler, C.R. Howell, J.H. Kelley, Krishichayan, R. Marci, G. Rusev, C. Ryan, S.A. Sheets, M.A. Stoyer, A.P. Tonchev, W. Tornow, D.J. Vieira, J.B. Wilhelmy, Nuclear Data Sheets 131, 319 (2016)

[4] H.R. Weller, M.H. Ahmed, H. Gao, W. Tornow, Y.K. Wu, M. Gai, and R. Miskimen, Progress in Particle and Nuclear Physics 62, 257 (2009)

[5] J.A. Grundl, D.M. Gilliam, N.D. Dudey, and R.J. Popek, Nuclear Technology 25, 237 (1975)

[6] www.canberra.com

[7] D.C. Radware, Nuclear Instruments and Methods in Physics Research Section A 361, 297 (1995) and www . radware . phy . ornl.gov

[8] J. Theuerkauf, S. Esser, S. Krink, M. Luig, N. Nicolay, O. Stuch, and H. Wolters, Program TV, Institute for Nuclear Physics, University of Cologne, 1993 (unpublished)

[9] H. Naik, F. Carrel, G.N. Kim, F. Laine, A. Sari, S. Normand, A. Goswami, Eur. Phys. J. A 49, 94 (2013) 